\title{
Separating the roles of electrons and holes in the organic magnetoresistance of aluminum tris(8-hydroxyquinoline) organic light emitting diodes
}

\author{
N. Rolfe, P. Desai, P. Shakya, T. Kreouzis, and W. P. Gillin ${ }^{\text {a) }}$ \\ Department of Physics, Queen Mary, University of London, Mile End Road, London E1 4NS, United \\ Kingdom
}

(Received 2 July 2008; accepted 27 August 2008; published online 20 October 2008)

\begin{abstract}
Measurements of the effect of a magnetic field on the photocurrent in an aluminum tris(8-hydroxyquinolate) based organic light emitting diode have shown that it is possible to identify the contribution to the organic magnetoresistance of both electrons and holes. For holes the effect of a magnetic field is to decrease the mobility, whereas for electrons the magnetic field appears to increase the mobility. These changes are suggested to be brought about through the magnetic field dependence of the scattering of electrons and holes with excited states within the device. (C) 2008 American Institute of Physics. [DOI: 10.1063/1.3000454]
\end{abstract}

\section{INTRODUCTION}

The effect of magnetic fields on the charge transport in organic light emitting diodes (OLEDs) has been attracting increasing interest. In 2003 Kalinowski et al. ${ }^{1}$ investigated the performance of OLEDs based on aluminum tris $(8$ hydroxyquinoliate $)\left(\mathrm{Alq}_{3}\right)$ OLEDs and found that the current through the device could be increased by $\sim 2.5 \%$ at a magnetic field density of less than $0.5 \mathrm{~T}$. They also found a simultaneous increase in efficiency of $\sim 3 \%$. The following year Francis et $a l^{2}$ showed that the effect of a magnetic field on the current in a device could also be seen in polymer systems, and they referred to it as magnetoresistance. They suggested that the effect was due to hole transport, despite the observation of electroluminescence from their devices. Mermer $e t ~ a l .^{3}$ went on to show that the effect could be seen in a range of polymer and small molecule systems and dubbed it organic magnetoresistance (OMR). Although the effect appears to be common in organic systems, there is still debate as to what the mechanism or mechanisms behind it are. Sheng et al. ${ }^{4,5}$ showed that the functional form of the effect was similar to that which could be expected from either hyperfine or spin-orbit interactions, although the mechanism through which these effects could influence the current transport was not clear.

More recently several models have been put forward to explain OMR. Prigodin et al. ${ }^{6}$ suggested that the effect may be due to changes in the electron-hole recombination rate induced by the magnetic field and hence altering the current in the device. Bobbert et l. $^{7}$ suggested that the effect of the magnetic field is to alter the ratio between polaron and bipolaron formation, and this affects the mobility of the charge carriers in the device. Desai et $_{\text {al. }}{ }^{8}$ suggested that the effect of the magnetic field is to alter the intersystem crossing rate between singlet and triplet states. This impacts on the current transport through the role of excited states as trapping centers for charge carriers. This model, and that of Prigodin et

\footnotetext{
a) aElectronic mail: w.gillin@qmul.ac.uk.
}

al., ${ }^{6}$ explains the observation that in most systems the onset of OMR seems to be intimately linked with light emission from devices. Desai et al. ${ }^{9}$ went on to show that the sign of the magnetoresistance could be changed by altering the thickness of the active layer in a device structure and that this change could be attributed to the role of exciton dissociation. This work showed that although the fundamental effect of the magnetic field was to alter the intersystem crossing rate between the singlet and triplet states, there were several mechanisms through which the exciton type and concentration could affect the current in a device.

In a recent work we investigated the effect of a magnetic field on the photocurrent in an $\mathrm{Alq}_{3}$ based OLED structure. ${ }^{10}$ This work was primarily designed to investigate whether photoinduced excitons could produce a magnetoresistance at voltages below the device turn-on voltage, where no magnetoresistance is measured in the dark. The work focused on the region from the open circuit voltage under illumination to the turn-on voltage $\left(V_{\mathrm{ON}}\right)$ of the device. In this work we have investigated the effect of a magnetic field on this photocurrent and we have shown that it is possible to isolate the contributions of both the electron and hole to the magnetoresistance.

\section{EXPERIMENTAL METHOD}

Devices were grown on indium tin oxide (ITO) coated substrates with a sheet resistivity of $\sim 13 \Omega / \square$ and consisted of $50 \mathrm{~nm}$ of $N, N^{\prime}$-diphenyl- $N, N^{\prime}$-bis(3methylphenyl)-(1, $1^{\prime}$-biphenyl)-4, $4^{\prime}$ diamine (TPD) as the hole transport layer and $90 \mathrm{~nm} \mathrm{Alq}_{3}$ as an emissive/electron transport layer with a cathode of $\operatorname{LiF}(1 \mathrm{~nm})+\mathrm{Al}$. The TPD and $\mathrm{Alq}_{3}$ were purchased from Aldrich and purified using train sublimation prior to use. The ITO substrate was patterned using photolithography and cleaned by ultrasonicating in detergent/water, acetone, and chloroform. Following this the ITO was treated in an oxygen plasma for $5 \mathrm{~min}$ at $30 \mathrm{~W}$ and 2.5 mbar pressure using a Diener Electronic Femto Plasma system. The plasma treated substrate was immedi- 


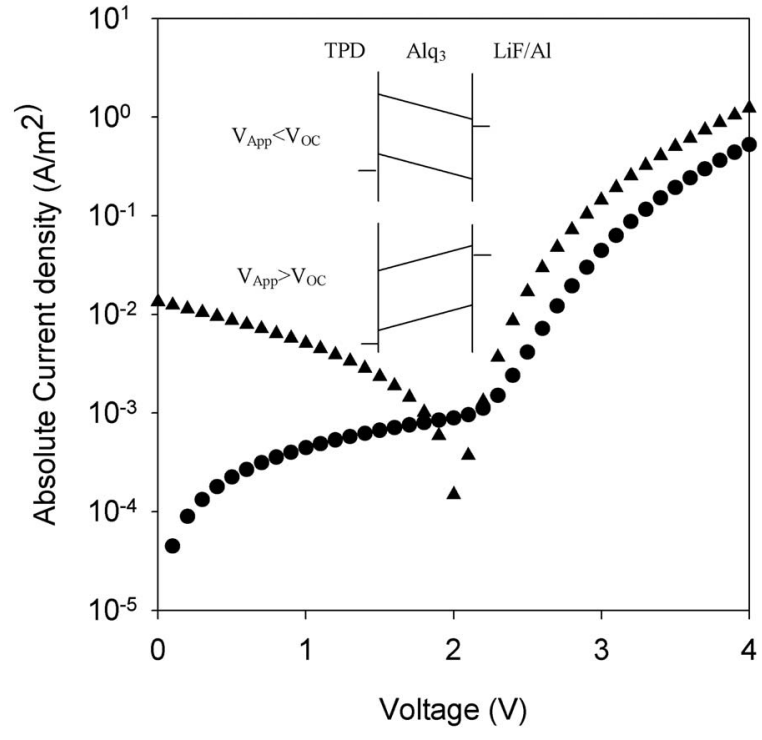

FIG. 1. The current-voltage characteristic for a $90 \mathrm{~nm} \mathrm{Alq}_{3}$ device with and without illumination. The inset shows a schematic of the band alignment in the $\mathrm{Alq}_{3}$ layer on either side of $V_{\mathrm{OC}}$.

ately transferred to the deposition chamber for device fabrication. The deposition of the organic layers and metal electrodes was performed using a Kurt J. Lesker SPECTROS evaporation system with a base pressure of $\sim 10^{-7}$ mbar during evaporation. The rate of deposition of organic materials was about $0.2 \mathrm{~nm} / \mathrm{s}$ while that of the metal was varied from $\sim 0.1$ to $1 \mathrm{~nm} / \mathrm{s}$. A calibrated oscillating quartz crystal monitor was used to determine the rate and thickness of the deposited layer. The whole device fabrication was performed without breaking vacuum. Final device areas were $\sim 4 \mathrm{~mm}^{2}$.

For the device measurements the samples were placed in a light tight sample holder under a vacuum of $<10^{-1}$ mbar. An array of five $395 \mathrm{~nm}$ LEDs, with a combined optical power output of $\sim 10 \mathrm{~mW}$, was placed directly above the devices to provide illumination for the photoconductivity measurements. Current-voltage measurements were recorded using a Keithley 236 source-measure unit. Before and after each measurement at an applied field, a corresponding measurement was made at null field, and these two null field measurements were averaged to give the baseline for determining the change in current with applied field. This method allowed us to remove any effects of sample drift from the measurements. For the field measurements the magnetic field was applied normal to the direction of current flow.

\section{RESULTS AND DISCUSSION}

Figure 1 shows the current-voltage characteristic for the $90 \mathrm{~nm} \mathrm{Alq}{ }_{3}$ device with and without illumination. The device shows an open circuit voltage under illumination of $\sim 2.1 \mathrm{~V}$ and a turn-on voltage of $\sim 2.2 \mathrm{~V}$. In an earlier paper ${ }^{9}$ we showed that in $\mathrm{ITO} / \mathrm{TPD} / \mathrm{Alq}_{3} / \mathrm{LiF} / \mathrm{Al}$ devices, the cathode was the dominant site for exciton dissociation. Given that for the excitation wavelength used in this work $(395 \mathrm{~nm})$ the absorption into TPD is considerably less than into $\mathrm{Alq}_{3}$, the majority of excitons formed in the device will be generated within the $\mathrm{Alq}_{3}$ layer. We can therefore consider the open

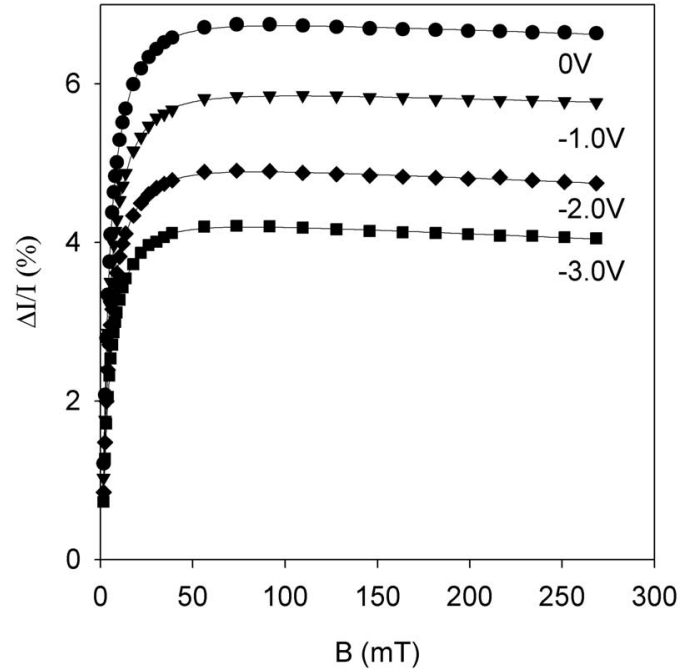

FIG. 2. The effect of a magnetic field on the photocurrent in a $90 \mathrm{~nm} \mathrm{Alq}_{3}$ device at voltages from 0 to $-3 \mathrm{~V}$. The solid lines are a guide to the eyes.

circuit voltage to be approximately where we have "flatbands" within the $\mathrm{Alq}_{3}$ layer. The inset to Fig. 1 therefore shows a schematic of the band alignment in the $\mathrm{Alq}_{3}$ layer at voltages above and below the open circuit voltage $V_{\mathrm{OC}}$. Therefore with excitons principally dissociating at the cathode, this means that for devices operated below $V_{\mathrm{OC}}$, the electric field in the device is such that the current in the device is predominantly carried by photogenerated holes; electrons generated at the cathode interface move directly into the cathode and, while contributing to the external current, they do not transit the device. Similarly between $V_{\mathrm{OC}}$ and $V_{\mathrm{ON}}$ the current in the device is predominantly carried by photogenerated electrons transiting the device; photogenerated holes generated at the cathode interface move directly into the cathode. Above $V_{\mathrm{ON}}$ both electrons and holes are injected from the cathode and anode, and hence the current in the $\mathrm{Alq}_{3}$ layer is bipolar.

Figure 2 shows the effect of a magnetic field on the photocurrent in the device at voltages between 0 and $-3 \mathrm{~V}$. The effect of the magnetic field is presented as $\Delta I / I(\%)$, which is the percentage change in current with applied field compared to the null field. For all voltages below $V_{\text {OC }}$, where the device is effectively in reverse bias, the current in the device is hole only and predominantly photogenerated. This photogenerated current is modulated by the magnetic field due to the perturbation in the intersystem crossing rate between the photogenerated singlets and the triplets, which due to their longer lifetime have a higher probability for dissociation than the singlets. The holes that are transiting the device can also interact with any excited states present in the device, and if there is a $B$ dependence to this interaction, this may also be observed in the $B$ dependence of the photocurrent. We have suggested ${ }^{8-11}$ that this interaction may be one of the causes of the OMR that has been observed in a number of organic systems. ${ }^{2,3}$ From Fig. 2 it can be seen that there is an initial rapid increase in the photocurrent, which tends to saturate at $\sim 50 \mathrm{mT}$. Similar effects have been seen in unilluminated $\mathrm{Alq}_{3}$ devices at low drive currents and is seen in the efficiency data, as a function of $B$ field, for $\mathrm{Alq}_{3}$ devices. ${ }^{9}$ 


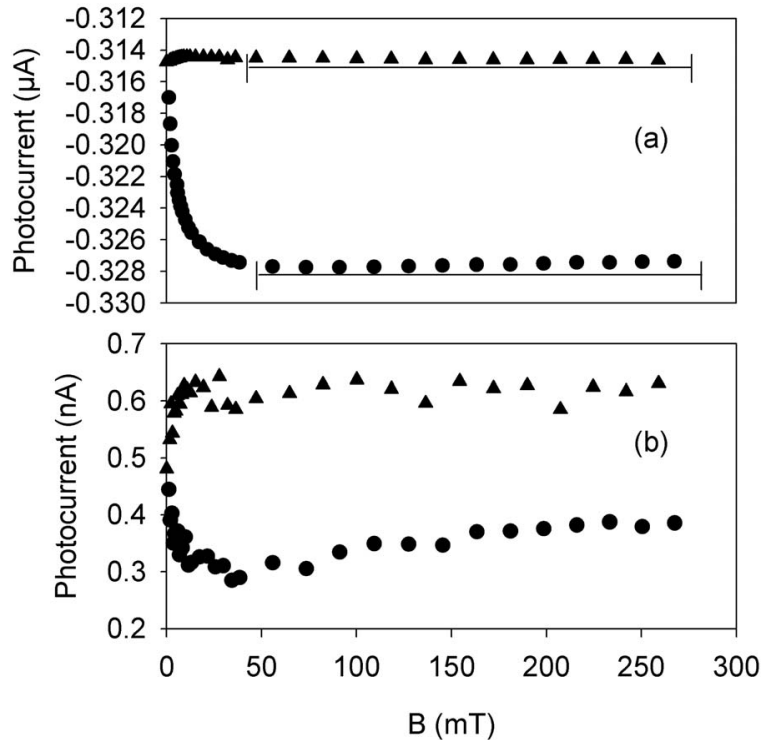

FIG. 3. The raw data for the current through a $90 \mathrm{~nm} \mathrm{Alq}$ device at $-3.0 \mathrm{~V}$ (a) and $2.105 \mathrm{~V}$ (b). The circles are the current with applied field and the triangles are the corresponding null field measurements. These null field measurements have been plotted against $B$ field solely to show the order in which the measurements were performed. The horizontal lines are provided to help see the small variation in photocurrent with applied magnetic field.

Above $\sim 50 \mathrm{mT}$ the photocurrent is seen to fall slightly with increasing $B$ field. This effect is small, $\sim 10^{-3} \% / \mathrm{mT}$, but is remarkably consistent, giving the same value at every measurement voltage from 0 to $-3 \mathrm{~V}$ in $0.1 \mathrm{~V}$ steps. Figure $3(\mathrm{a})$ shows the raw data for the measurement at $-3.0 \mathrm{~V}$. The null field measurements show that the device exhibits excellent stability during the measurement. It can be seen that the main effect of the magnetic field is to increase the magnitude of the photocurrent (making it more negative), and this effect saturates by $\sim 50 \mathrm{mT}$. This is very similar to the OMR behavior of unilluminated $\mathrm{Alq}_{3}$ devices. ${ }^{8-11}$ From Fig. 3(a) it can also be seen that above $\sim 50 \mathrm{mT}$, the increasing $B$ field starts to produce a small decrease in the magnitude of the photocurrent, compared to that at $50 \mathrm{mT}$.

For unilluminated $\mathrm{Alq}_{3}$ devices we have seen that while the efficiency data saturate at $B$ fields above $\sim 50 \mathrm{mT}$, the OMR data, particularly at high current densities, tend to continue increasing. ${ }^{9}$ We have suggested that this continued increase in the current with increasing magnetic field could be due to a $B$ field dependence of the interaction strength between the charge carrier and triplets. For OMR data this additional increase in the current at high field suggested that the interaction strength was getting weaker with increasing $B$ field, resulting in an increase in mobility. However, from the OMR data it was not possible to distinguish the contribution from electrons and holes. From these photocurrent data however, where the predominant charge carriers transiting the device are holes, the fact that the magnitude of the current decreases suggests that for holes in $\mathrm{Alq}_{3}$ the interaction strength increases with increasing $B$ field, decreasing the hole mobility.

Figure 4 shows the effect of a magnetic field on the device current at voltages between $V_{\mathrm{OC}}$ and $V_{\mathrm{ON}}$. Just above $V_{\mathrm{OC}}$ the change in current with applied field appears to satu-

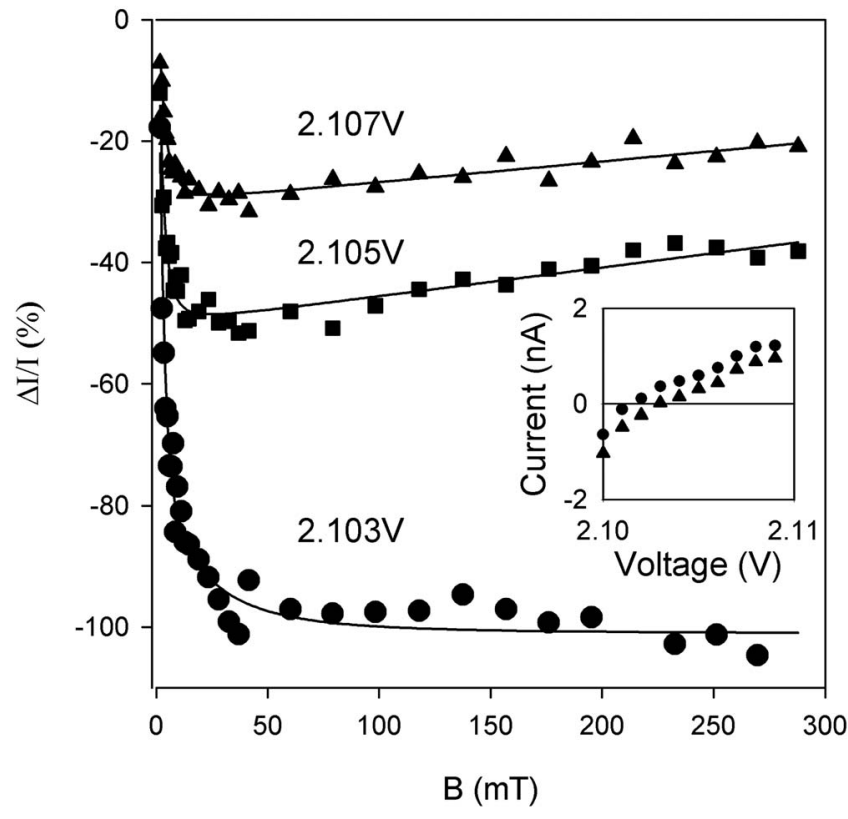

FIG. 4. The effect of a magnetic field on the photocurrent in a $90 \mathrm{~nm} \mathrm{Alq}_{3}$ device at voltages just above $V_{\mathrm{OC}}$. The solid lines are a guide to the eyes. The inset shows the change in the $I V$ curve around $V_{\mathrm{OC}}$ with (triangles) and without (circles) an applied field of $28 \mathrm{mT}$.

rate and has a form similar to that observed for the change in efficiency for all group III quinolate devices. For devices operating below $V_{\mathrm{ON}}$, the current is predominantly photogenerated and the source of the current is the same. However, in this regime the electric field in the device causes electrons to transit the device while holes move straight into the cathode.

As the effect of the applied $B$ field is to create more photogenerated carriers, it is necessary to address the fact that in this regime, between $V_{\mathrm{OC}}$ and $V_{\mathrm{ON}}$, the measured OMR is actually negative. The reason for this can be seen in the inset to Fig. 4, which shows the $J V$ curve for the device around $V_{\mathrm{OC}}$ both with and without an applied $B$ field. It can be seen that at a constant voltage, the photocurrent decreases, which appears to be contradictory. However, the negative OMR, which is implied by this apparent decrease in photocurrent, is an artifact of the fact that we are performing the experiment at constant voltage. The actual effect of the increased exciton dissociation with applied $B$ field is to produce a small increase in $V_{\mathrm{OC}}(\sim 1 \mathrm{mV})$.

As the applied voltage is increased, it can be seen that in addition to the rapid negative change in current that saturates at $\sim 50 \mathrm{mT}$, there is also an increase in the current that occurs at $>50 \mathrm{mT}$. Given that in this region the predominant charge carriers are electrons, we suggest that this component may be the magnetic field dependence of the interaction between the electrons and the excited states present in the device. In contrast to what was found for the holes below $V_{\mathrm{OC}}$, this component increases the absolute current [see Fig. 3(b)] in the device and, if it is due to the magnetic field dependence of the electron-triplet interaction, suggests that the interaction strength decreases with applied magnetic field and increases the electron mobility.

At voltages above $2.15 \mathrm{~V}$ the OMR of the device under 
illumination looks identical in shape to that of an unilluminated device at a high voltage $(>2.7 \mathrm{~V})$. For the unilluminated device no OMR can be observed until voltages of $>2.5 \mathrm{~V}$, although it is not until $2.7 \mathrm{~V}$ that the noise on the OMR data is sufficiently small to be able to resolve a shape to the curve. (At $2.5 \mathrm{~V}$ the differences between the null field and applied field measurements are consistently positive but the changes are very small and there is a lot of noise in the data.) We have suggested that the fact that no OMR can be seen in these devices at voltages below the turn-on voltage is an evidence that the OMR is linked to the presence of excitons in the device. For the unilluminated device the electron injection when the device first switches on is very low, as evidenced by the lack of electroluminescence from the device. However, when the device is illuminated the electrons released at the cathode due to the dissociation of photogenerated excitons are free to recombine with injected holes, and these excitons are the ones that generate the OMR response.

\section{CONCLUSIONS}

We have shown that it is possible to differentiate the contribution of electrons and holes to OMR by operating an OLED device under illumination and changing the bias conditions. In reverse bias it can be seen that there is a $B$ dependence to the interaction between a hole and an excited state and that the interaction strength appears to increase with increasing magnetic field. Above the open circuit voltage the interaction is predominantly between electrons and the excited states, and the interaction strength for this process appears to decrease with increasing magnetic field. The magnitude of the electron-exciton interaction is much greater than that for holes, and as they operate in opposite directions it suggests that for $\mathrm{OMR}$ in $\mathrm{Alq}_{3}$ devices, it is the electronexciton interaction that dominates.

The technique of using magnetophotoconductivity measurements to study carrier-exciton interactions has wider applications as it is possible to control the carrier type transiting a device through control of the interfaces. For example we have already demonstrated that the introduction of a 2,9dimethyl-4,7-diphenyl-1,10-phenanthroline (BCP) interface layer between $\mathrm{Alq}_{3}$ and the cathode greatly reduces the exciton dissociation at this interface, and by choosing an anode interface material that encourages exciton dissociation, one could make electrons the predominant carrier species in the reverse bias region. Thus it would be much easier to study the effect of magnetic fields on different charge carriers.

${ }^{1}$ J. Kalinowski, M. Cocchi, D. Virgili, P. Di Marco, and V. Fattori, Chem. Phys. Lett. 380, 710 (2003).

${ }^{2}$ T. L. Francis, O. Mermer, G. Veeraraghavan, and M. Wohlgenannt, New J. Phys. 6, 185 (2004).

${ }^{3}$ O. Mermer, G. Veeraraghavan, T. L. Francis, and M. Wohlgenannt, Solid State Commun. 134, 631 (2005)

${ }^{4}$ Y. Sheng, T. D. Nguyen, G. Veeraraghavan, O. Mermer, M. Wohlgenannt, S. Qiu, and U. Scherf, Phys. Rev. B 74, 045213 (2006).

${ }^{5}$ Y. Sheng, T. D. Nguyen, G. Veeraraghavan, O. Mermer, and M. Wohlgenannt, Phys. Rev. B 75, 035202 (2007).

${ }^{6}$ V. N. Prigodin, J. D. Bergeson, D. M. Lincoln, and A. J. Epstein, Synth. Met. 156, 757 (2006).

${ }^{7}$ P. A. Bobbert, T. D. Nguyen, F. W. A. van Oost, B. Koopmans, and M. Wohlgenannt, Phys. Rev. Lett. 99, 216801 (2007).

${ }^{8}$ P. Desai, P. Shakya, T. Kreouzis, W. P. Gillin, N. A. Morley, and M. R. J. Gibbs, Phys. Rev. B 75, 094423 (2007).

${ }^{9}$ P. Desai, P. Shakya, T. Kreouzis, and W. P. Gillin, J. Appl. Phys. 102, 073710 (2007).

${ }^{10}$ P. Desai, P. Shakya, T. Kreouzis, and W. P. Gillin, Phys. Rev. B 76, 235202 (2007).

${ }^{11}$ P. Shakya, P. Desai, M. Somerton, G. Gannaway, T. Kreouzis, and W. P. Gillin, J. Appl. Phys. 103, 103715 (2008). 